\title{
Detección de Norovirus GI Y GII en muestras de agua del Río Piura mediante la técnica de RT-PCR en tiempo real
}

\author{
Detection of Norovirus GI and GII in water samples from the Piura River using the RT-PCR technique in \\ real time
}

\author{
Arabela Ritchie ${ }^{1}$, Armando Hung ${ }^{1}$, Muriel Gómez-Sánchez ${ }^{2}$
}

\section{RESUMEN}

Los Norovirus (NoV) son una de las causas más frecuentes de infecciones gastrointestinales agudas en humanos y un problema de salud importante en países en desarrollo. El objetivo del estudio fue detectar la presencia del Norovirus GI y GII en muestras de agua del Rio Piura mediante la concentración del virus en agua y RT-PCR en tiempo real. Para ello se recolectaron muestras de agua en botellas estériles en tres (3) puntos determinados del río, cada 15 días, desde mayo hasta Setiembre del 2013. Se obtuvo 20.8\% (5/24) de muestras positivas para Norovirus GI mediante la técnica de RT-PCR en tiempo real. Ninguna de las muestras dio positivo a Norovirus GII. Este es el primer reporte de Norovirus GI en muestras de agua del Rio Piura. Los métodos propuestos podrían ser utilizados como herramienta para la investigación de patógenos en aguas del Rio Piura y la Bahía de Sechura.

PALABRAS CLAVE: Norovirus GI y GII, RT--PCR en tiempo real, río Piura.

\section{SUMMARY}

Norovirus (NoV) is a leading cause of acute gastroenteritis in humans and a major health problem in developing countries. The aim of this study was to detect the presence of Norovirus GI and GII in water samples of the Piura River by viral concentration and real time RT-PCR. Water samples, were collected in 3 specific points of the river in sterile bottles fortnightly from May to September 2013. Our results indicate that 20.8\% (5/24) of the samples were positive for Norovirus G1 by real time RT-PCR. None of the samples tested positive for Norovirus GII. This is the first report of the presence of Norovirus GI in Piura River waters. The proposed method can be used as a tool to enhance research of pathogens in the Bay area water and Piura River water.

KEY WORDS: Norovirus GI and GII, real time RT-PCR, Piura river.

\section{INTRODUCCIÓN}

Los Norovirus (NoV) se encuentran entre los enteropatógenos más comunes en todo el mundo, siendo una causa importante de diarrea infantil en países en desarrollo (Patel et al., 2008). Son miembros de la familia Caliciviridae y pueden ser subdivididos en 5 genogrupos, siendo los genogrupos GI y GII responsables de la mayoría de los casos clínicos, los cuales son clasificados como grupo 2 de agentes biológicos por la Unión Europea (2004), y como grupo de riesgo 2 de agentes etiológicos humanos por el Instituto Nacional de Salud de Estados Unidos (Koo, Ajami, Atmar, y DuPont, 2010; Zheng et al., 2006).

Facultad de Medicina Veterinaria y Zootecnia, Universidad Peruana Cayetano Heredia. Lima, Perú.

Organismo Nacional de Sanidad Pesquera. Lima, Perú. 
La sintomatología abarca vómitos de aparición aguda, diarrea acuosa sin sangre, calambres abdominales y náuseas.

Afectan a individuos de todas las edades y son responsables de hasta 200,000 muertes de niños menores de 5 años en países en desarrollo (Patel et al., 2008). El período de incubación es de 24-48 horas y se transmite a través de la ruta fecal-oral (Moe, Christmas, Echols, \& Miller, 2001) por lo que se consideran altamente contagiosos. El genotipo GII/4 constituye el más prevalente de los 46 genotipos que existen alrededor del mundo. En Brasil se reportaron brotes donde el $72.5 \%(50 / 80)$ fueron por este genotipo, en Vietnam el $78 \%$ de brotes estuvo relacionado al mismo genotipo (53/68) (Hansman et al., 2004) y en Japón se reportaron 39 brotes entre diciembre de 2001 y abril de 2006 (Fukuda, Sasaki, Takao, y Seno, 2008). La epidemiología de las infecciones por NoV se han subestimado por mucho tiempo debido a la limitada disponibilidad de métodos de detección y a la incapacidad para cultivar los NoV en líneas celulares. Como consecuencia de un aumento en el uso de técnicas moleculares, se ha logrado detectar NoV en aguas superficiales, aguas subterráneas, ríos, entre otros (Haramoto, Katayama, Oguma, y Ohgaki, 2005; Maunula, Miettinen, y Von Bonsdorff, 2005). Los diferentes genogrupos de Norovirus; en especial el GII están dispersos en aguas de desagüe tratadas y sin tratar (Lodder et al., 1999; Van den Berg, Lodder, van der Poel, Vennema, y de Roda Husman, 2005) que pueden llevar a la contaminación de agua de consumo humano con efectos adversos en la salud humana.

A pesar de que las estadísticas en países en desarrollo son escasas, las infecciones por NoV parecen ser comunes. Por ejemplo, en el Perú, se detectó por medio de PCR en el 17\% de los pacientes pediátricos hospitalizados en el Instituto Nacional del Niño con gastroenteritis negativas a otros patógenos (Parashar et al, 2004). Además, se ha reportado un brote por $\mathrm{NoV}$ GI en un buque naval de EE.UU. después de haber visitado Perú, donde 130 de 230 tripulantes estuvieron infectados (Gonzaga, Ramos, Maves, Freeman y Montgomery, 2011). Un estudio realizado por Saito et al. (2014), concluyó que la infección por NoV en Perú ocurre a temprana edad, en un año de seguimiento a 220 niños el $80 \%$ presentó infecciones relacionadas a múltiples genotipos; y en dos años de seguimiento a $189,71 \%$ presentó infecciones por NoV.

Muestras de agua obtenidas de fuentes medioambientales suelen contener bajas concentraciones de virus patogénicos. Actualmente se utilizan diferentes métodos para concentrar las muestras y lograr aislar y detectar de manera eficiente los virus (Gregory, Litaker y Noble, 2006; Atmar et al., 1995; Da Silva et al., 2007). Katayama et al. (2002) desarrollaron un método empleando membranas con cargas negativas, cationes y soluciones ácidas, de manera simple y menos costosa en comparación a los métodos de concentración con extracto de carne o resinas (Abbaszadegan, Huber, Gerba, y Pepper, 1993).

La detección de NoV se basa en la amplificación del genoma utilizando técnicas biomoleculares como; la reacción en cadena de polimerasa- transcriptasa reversa en tiempo real (RT-PCR). El tiempo, precisión, eficiencia y sensibilidad del método son de gran importancia y los avances en la detección de virus en agua han puesto a esta herramienta en una nueva perspectiva para la clasificación sanitaria de las aguas y áreas de cultivo de moluscos bivalvos alrededor del mundo.

La bahía de Sechura en el departamento de Piura es uno de los centros de repoblamiento de moluscos bivalvos más importantes del país; en especial para la Concha de abanico (Argopecten purpuratus). Sin embargo, en los últimos años, numerosos casos de enteropatógenos virales como Hepatitis A han sido asociados a la importación de moluscos bivalvos peruanos en países de la Comunidad Europea (Polo, Vilariño, Manso, y Romalde, 2010), generando importantes pérdidas económicas asociadas a la restricción de la exportación y la pérdida de puestos de trabajo. Dentro de las medidas correctivas para superar esta restricción, se encuentra determinar las principales fuentes de contaminación de los bancos naturales de bivalvos, así como sus zonas de repoblamiento. Esta información, ayudaría a las autoridades establecer planes de control y prevención para evitar la contaminación de las zonas de cultivo y repoblamiento.

\section{MATERIAL Y MÉTODOS}

Las muestras de agua empleadas fueron provenientes del río Piura, provincia de Sechura (situada en la Costa Norte del Perú a $54 \mathrm{~km}$ de la ciudad de Piura.), Departamento de Piura. La cuenca del río Piura está situada geográficamente entre los paralelos $4^{\circ} 42^{\prime}$ y $5^{\circ} 45^{\prime}$ de latitud sur y los meridianos $79^{\circ} 29^{\prime}$ y $81^{\circ}$ de longitud oeste. 
Las muestras de agua se recolectaron quincenalmente durante los meses de mayo a setiembre de 2013. Los tres puntos de referencia para la toma de muestra se reconocieron mediante coordenadas de GPS previamente seleccionadas. El primer punto fue el brazo sur de la bifurcación del río $\left(5^{\circ} 33^{\prime} 17.626^{\prime \prime} \mathrm{S}\right.$, $\left.80^{\circ} 49^{\prime} 35.481^{\prime \prime} \mathrm{W}\right)$, el segundo punto fue la zona central a nivel del puente del río Piura $\left(5^{\circ} 33^{\prime} 02.179^{\prime \prime}\right.$, $\left.80^{\circ} 49^{\prime} 09.139^{\prime \prime} \mathrm{W}\right)$, y el tercer punto la bifurcación norte del rio $\left(5^{\circ} 33^{\prime} 04.153^{\prime \prime} \mathrm{S}, 80^{\circ} 49^{\prime} 26.826^{\prime \prime} \mathrm{W}\right)$.

En total se recolectaron 24 muestras. Para la recolección de muestras de agua se utilizó baldes previamente desinfectados y atados a una cuerda. El agua se almacenó en botellas de plástico de 1 litro, esterilizadas y rotuladas. El transporte de las muestras se realizó en contenedores con hielo. Las muestras se analizaron en el laboratorio de Biología Molecular de la Facultad de Medicina Veterinaria y Zootecnia de la Universidad Peruana Cayetano Heredia en Lima Perú.

El método que se utilizó para la concentración de virus se denomina Baylor (Atmar et al., 1995; Da Silva et al., 2007) y ha sido adaptado de Marcos, GómezSánchez, y Hung (2014). El primer paso consistió en añadir $10 \mathrm{ml}$ de solución de polietilenglicol (PEG) 8000 al 50\% a $40 \mathrm{ml}$ de muestra de agua, la cual se dejó en agitación a $4{ }^{\circ} \mathrm{C}$ durante una noche. Se centrifugó la mezcla a 4000 revoluciones por minuto (RPM) por una hora y media a $4^{\circ} \mathrm{C}$. Este paso tuvo como función la precipitación de los virus. El pellet obtenido se disolvió en $3 \mathrm{ml}$ de agua estéril, con $1 \mathrm{ml}$ de tampón PK $4 X, 40 \mu l$ de proteinasa $\mathrm{K}(20 \mathrm{mg} / \mathrm{ml})$, y $4 \mathrm{ml}$ de fenol:cloroformo:agua en proporción de 68:18:14. La mezcla se homogenizó por 1 minuto y se centrifugó a
4000 RPM por 5 minutos a una temperatura de $4^{\circ} \mathrm{C}$. A continuación, se añadió a la fase superior $4 \mathrm{ml}$ de cloroformo:alcohol isoamílico en proporción de 24:1, y se homogenizó por 1 minuto. Se volvió a centrifugar a $4000 \mathrm{RPM}$ por 5 minutos a $4^{\circ} \mathrm{C}$.

A la fase acuosa obtenida se le añadió $400 \mu \mathrm{l}$ de acetato de sodio $3 \mathrm{M}$ y $8 \mathrm{ml}$ de etanol absoluto previamente refrigerado. Se dejó precipitar por 30 minutos y se centrifugó a 4000 RPM por 30 minutos a $4^{\circ} \mathrm{C}$. Se descartó el sobrenadante y se disolvió el pellet obtenido con $4.5 \mathrm{ml}$ de agua estéril a una temperatura de $56^{\circ} \mathrm{C}$. Se añadió $1.8 \mathrm{ml}$ de bromuro cetil trimetil amonio y después de 15 minutos de incubación a temperatura ambiente, se centrifugó a $25^{\circ} \mathrm{C}$ por 30 minutos a 4000 RPM. Se volvió a suspender el pellet en $150 \mu \mathrm{l}$ de solución salina, $30 \mu \mathrm{l}$ de acetato de sodio $3 \mathrm{M}$ y $900 \mu \mathrm{l}$ de etanol absoluto frío y se agitó por inversión. Luego, se precipitó por 30 minutos y se centrifugó a $4^{\circ} \mathrm{C}$ por 30 minutos a 4000 RPM. Se añadieron $500 \mu \mathrm{l}$ de etanol al $70 \%$ frío y se centrifugó a $4^{\circ} \mathrm{C}$ por 30 minutos a $4000 \mathrm{RPM}$. El pellet final se secó y volvió a suspender en $100 \mu 1$ de agua estéril.

Para la extracción del ARN viral en agua concentrada se usó el kit comercial QIAamp viral RNA mini kit (QIAGEN-ALEMANIA) y se procedió de acuerdo al protocolo del fabricante. Para la detección de la presencia de Norovirus por RT-PCR en tiempo real se empleó el kit comercial RNA Ultrasense OneStep Quantitative RT-PCR System (INVITROGEN) y se siguió las especificaciones del fabricante. El Norovirus GI se amplificó utilizando el primer forward QNIF4 (FW) CGC TGG ATG CGN TTC CAT (Da Silva et al., 2007), el primer reverse NV1LCR (REV) CCT TAG ACG CCA TCA TCA TTT AC y la sonda

Tabla 1. Resultados de la detección del genoma de Norovirus I y Norovirus II en muestras de agua del Río Piura (Mayo a Setiembre del 2013).

\begin{tabular}{ccccccc}
\hline \multirow{2}{*}{$\begin{array}{c}\text { Fecha de } \\
\text { muestreo }\end{array}$} & \multicolumn{7}{c}{ Rama Norte } & \multicolumn{2}{c}{ Puente } & \multicolumn{2}{c}{ Rama Sur } \\
\cline { 2 - 6 } & Norovirus I & Norovirus II & Norovirus I & Norovirus II & Norovirus I & Norovirus II \\
\hline 14 de mayo & - & - & $(+)$ & - & $(+)$ & - \\
07 de junio & - & - & - & - & $(+)$ & - \\
21 de junio & - & - & $(+)$ & - & - & - \\
05 de julio & - & - & - & - & - & - \\
19 de julio & - & - & - & - & - & - \\
09 de agosto & - & - & - & - & - & - \\
23 de agosto & - & - & - & - & - \\
23 de agosto & - & - & - & - & - \\
\hline
\end{tabular}


NVGG1p (PROBE) TGG ACA GGA GAY CGC RAT CT (Svraka et al., 2007). El Norovirus GII se amplificó utilizando el primer forward QNIF2 (FW) ATG TTC AGR TGG ATG AGR TTC TCW GA (Loysi et al., 2005), el primer reverse COG2R (REV) TCG ACG CCA TCT TCA TTC ACA (Kageyama et al, 2003) y la sonda QNIFs (Probe) AGC ACG TGG GAG GGC GAT CG (Loysi et al., 2005). Para cada reacción se preparó una mezcla maestra bajo frío con un volumen final de 25 ul (FW 500nM, REV 900nM, probe $250 \mathrm{Nm}$ ).

La programación del termociclador en el LightCycler 480 , tuvo un paso inicial de $55^{\circ} \mathrm{C}$ por 1 hora, $95^{\circ} \mathrm{C}$ por $5 \mathrm{~min}$, seguido por 45 ciclos a $95^{\circ} \mathrm{C}$ por 15 segundos, $60^{\circ} \mathrm{C}$ por 1 minuto y $65^{\circ} \mathrm{C}$ por un minuto.

Los datos fueron analizados por el software del termociclador LightCycler 480 (software reléase
1.5.2.62). La información que muestra el programa toma como base la cuantificación viral en cada muestra (Cp), previa cuantificación absoluta de lenticulas (CEFAS) de NoV GI y NoV GII.

\section{RESULTADOS}

En $20,8 \%(n=5)$ de las muestras analizadas se detectó el genoma de Norovirus GI por la prueba de RT-PCR en tiempo real. Se obtuvo 72,60 copias de ARN/ $\mu 1(\mathrm{Cp}: 35,78)$ en la muestra del punto Sur $(14$ de mayo del 2013), 93,47 copias de ARN/ $\mu$ l (Cp: 35,42 ) en la muestra del punto Puente (14 de mayo del 2013), 33,07 copias de ARN/ $\mu 1$ (Cp: 36,9) en la muestra del punto Sur (07 de junio del 2013), 78,42 copias de ARN/ $\mu \mathrm{l}(\mathrm{Cp}: 35,67)$ en la muestra del punto Puente (28 de junio del 2013), y 72,6 copias de ARN/ $\mu \mathrm{l}(\mathrm{Cp}: 35,78$ ) en la muestra del punto Sur (28 de junio del 2013) No se obtuvo amplificación para Norovirus GII, en ninguna de las muestras (tablas 1 y 2 ; figura 1).

Tabla 2: Cuantificación de la carga viral seriada en muestras positivas a Norovirus GI

\begin{tabular}{|c|c|c|c|}
\hline $\begin{array}{r}\text { Muestras positivas } \\
\text { GI* }\end{array}$ & Norovirus & Ciclo de cuantificación & Cuantificación Copias de ARN/ $\mu 1$ \\
\hline 1 & & 35.78 & 72.60 \\
\hline 2 & & 35.42 & 93.47 \\
\hline 3 & & 36.9 & 33.07 \\
\hline 4 & & 35.67 & $7-8.42$ \\
\hline 5 & & 35.78 & 72.60 \\
\hline
\end{tabular}

*Muestra del río Piura del punto sur del 14 de mayo del 2013 (1), del punto puente del 14 de mayo del 2013 (2), del punto sur del 07 de junio del 2013 (3), del punto Puente del 28 de junio del 2013 (4) y del punto Sur del 28 de junio del 2013 (5)

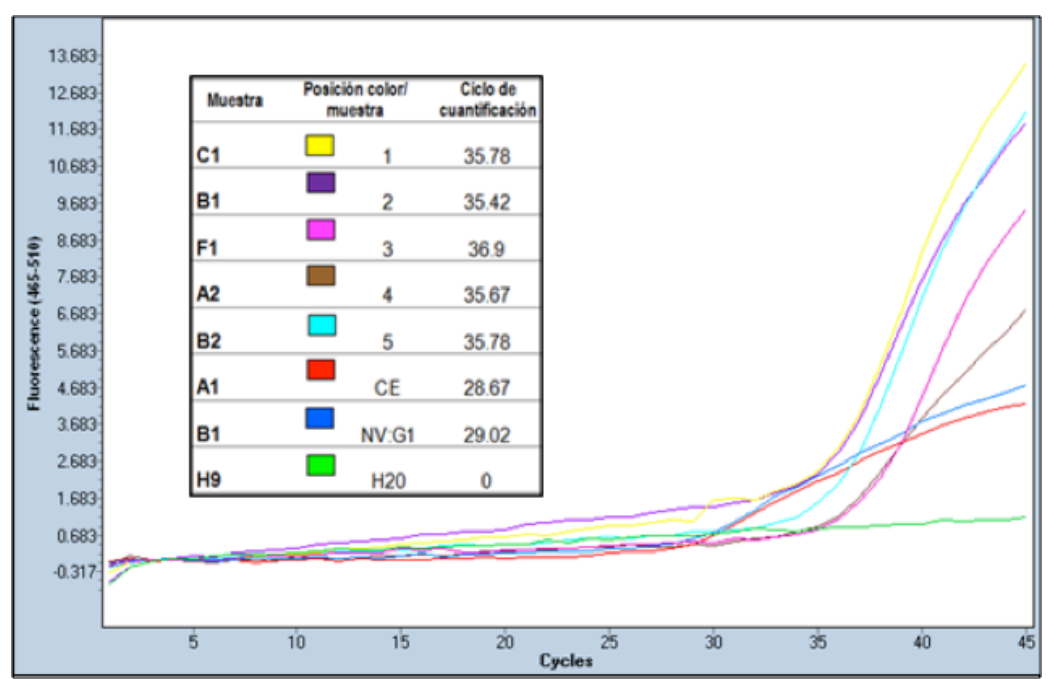

Figura. 1. RT-PCR en tiempo real de muestras positivas a Norovirus GI. Leyenda: Control negativo: Verde, control de extracción positivo Azul; control externo de RNA de lenticula $(+)$ Rojo. Las muestras (1-5) dan relación a las muestras del cuadro 3; positivas a Norovirus GI. 


\section{DISCUSIÓN}

El estudio encuentra resultados positivos irregulares en los diferentes muestreos, lo que indicaría que la detección del NoV GI podría ser mayor de considerar muestras repetidas en cada uno de los muestreos, lo que mejoraría la capacidad de detección del virus. $\mathrm{La}$ ausencia de muestras positivas a NoV GII en las muestras del estudio y los resultados irregulares de NoV GI, recalcan la necesidad de un estudio con un mayor número de muestras.

El porcentaje de muestras positivas para NoV GI $(20,8 \%)$ es mayor a los reportados por Wyn-Jones et al. (2011) en aguas recreacionales de Europa que fue del 9,4\% (136/141) pero en general más bajos que los encontrados por Maunula et al. (2005) en Finlandia que fue del 13\% (18/41); por Victoria et al. (2014) en el río Uruguay, que fue del 51\% (49/96) y por Kitajima, Haramoto, Phanuwan, Katayama, y Ohgaki (2009) en el río Tamagawa, Japon que fue del 31\% (15/48). La cuantificación de la carga viral de las muestras positivas fueron más elevadas que las obtenidas por Hewitt et al. (2007) en Nueva Zelanda (1080 +/- 569) NoV GI v/L; y por Westrell et al. (2006) en el río Meuse, $(1700 \mathrm{v} / \mathrm{L})$.

La presencia de Norovirus GI en aguas del río Piura representa un riesgo para la salud pública debido a que la población utiliza diariamente sus aguas, así como a las áreas de maricultura y en general la bahía a donde fluye el río. Los virus detectados por RT-PCR en tiempo real deben estar activos y la técnica nos permite discernir de patógenos nos activos. Durante los muestreos que se realizaron en los tres puntos, es notoria la presencia de residuos de diferentes tipos; domésticos (restos de comida, pañales, papeles, botellas), industriales (valvas de conchas de abanico, residuos de pescado, etc.) ganadería (crianza de cerdos en las ribera del río, deposiciones de vacas y caballos) así como la presencia de niños jugando en el río y personas lavando cajas de plástico y sistemas de cultivo de concha de abanico; todas posibles fuentes de contaminación.

Desafortunadamente el monitoreo de Norovirus en alimentos y agua usualmente se lleva a cabo cuando se sospecha de una posible contaminación de los mismos. En esta etapa es más difícil reducir la transmisión y puede dar inicio a un brote. Actualmente, solo una pequeña fracción de los alimentos es monitoreada con técnicas como RT-PCR en tiempo real. Un elevado número de infecciones por Norovirus, ocurren como consecuencia de trabajadores infectados sintomática o asintomáticamente y que manipulan alimentos (Ozawa, Oka, Takeda, y Hansman, 2007). La carga socio económica que un brote representa en la población es alta y la morbilidad también.

La bahía de Sechura, constituye uno de los bancos naturales de concha de abanico más importante del país y de acuerdo a un estudio de Cavero y Rodríguez (2008), es también un importante centro de repoblamiento y cultivo de moluscos bivalvos. La presencia de Norovirus GI en el agua de río Piura representa un riesgo potencial para todas las actividades que se desarrollan en su entorno, pues fluye directamente a la bahía. Se debe de tener en consideración que la infección con NoV ocurre con frecuencia después del consumo de alimentos susceptibles a la contaminación con deshechos de heces humanas como moluscos bivalvos y ostras (De Keuckelaere, Li, Deliens, Stals, y Uyttendaele, 2015; Sarvikivi et al., 2012; Brassard, Gagné, Généreux y Côté, 2012; Le Guyader, Atmar, y Le Pendu, 2012; Alfano-Sobsey et al., 2012). Actualmente, la clasificación sanitaria de la bahía en las áreas denominadas Matacaballo, Constante y Las Delicias es de tipo A y en las playas de Chulliyachi, San Pedro y San Pablo de tipo B. Esta clasificación se basa solo en el criterio microbiológico y limita la comercialización de productos marinos sin pasar un proceso de depuración o tratamiento térmico (Regulación UE 853/2004).

Actualmente rige una restricción a la importación de moluscos bivalvos no pectínidos como las Palabritas o Coquinas (Donax sp) del Perú, por la detección de virus de hepatitis $\mathrm{A}$, considerándose que no cumplen los requisitos sanitarios exigidos por la legislación UE 2008/866/CE de la Unión Europea (2008). Este investigación revela la presencia de NoV en las aguas del río Piura, por lo que se hace necesario considerar dentro de los riesgos para la actividad de extracción de moluscos bivalvos en general, el manejo sanitario del río. Por otro lado, repetir este estudio en muestras de la Bahía y en otros puntos del Río Piura sería conveniente para determinar el origen de la contaminación y la presencia de NoV en las zonas de maricultura.

En la provincia de Sechura, la gestión de aguas servidas es inadecuada, la cultura y educación ambiental es escasa, la población no segrega los residuos sólidos y muchas veces los deposita en lugares no apropiados. El vertimiento de aguas servidas al río, en muchos casos directo y sin tratamiento, ha generado un aumento en la contaminación y ocasionado problemas 
sanitarios, que a su vez limitan en gran manera el desarrollo económico y la realización de actividades de maricultura en la bahía.

Debido al bajo número de partículas virales necesarias para causar enfermedad (Teunis et al., 2008), es importante manejar métodos eficientes y rápidos para recuperar los virus los cuales pueden ser usados para análisis de rutina e investigaciones de brotes. Para la recuperación de virus del agua un método optimo debe cubrir ciertos criterios; debe ser rápido y fácil de realizar, tener un alta eficiencia de recuperación y debe ser aplicable para concentrar un gran rango de virus, proveer un pequeño volumen de concentrado, bajo en costo, capaz de procesar amplios volúmenes de agua, ser repetido en el laboratorio y reproducible entre laboratorios (Block y Schwartzbrod, 1989).

Este estudio reporta por primera vez en el país la presencia de Norovirus GI en muestras de agua del río Piura, siendo de importancia sanitaria, social y económica para la acuicultura local, por lo que es necesario que las autoridades políticas de las diferentes entidades del Gobierno tomen medidas en forma conjunta para evitar la contaminación del ecosistema acuícola.

\section{CONCLUSIONES}

El 20,8\% (5) y el 0\% (0) de las muestras analizadas resultaron positivas a Norovirus G1 y GII por la prueba de RT-PCR en tiempo real.

La investigación reporta por primera vez la presencia del Norovirus GI en muestras de agua de río en el Perú, lo que representa información que permite valorar el riesgo de contaminación en moluscos bivalvos cultivados en las zonas cercanas a la desembocadura del Río Piura.

\section{Correspondencia}

Arabela Ritchie Martínez

Correo electrónico: arabela.ritchiemartinez@wur.nl

\section{REFERENCIAS BIBLIOGRÁFICAS}

1. Abbaszadegan, M., Huber, M. S., Gerba, C. P., \& Pepper, I. L. (1993). Detection of enteroviruses in groundwater with the polymerase chain reaction. Applied and Environmental Microbiology, 59(5), 1318-1324.

2. Alfano-Sobsey, E., Sweat, D., Hall, A., Breedlove, F.,
Rodriguez, R., Greene, S., ... \& Ledford, S. L. (2012). Norovirus outbreak associated with undercooked oysters and secondary household transmission. Epidemiology \& Infection, 140(2), 276-282.

3. Atmar, R. L., Neill, F. H., Romalde, J. L., Le Guyader, F., Woodley, C. M., Metcalf, T. G., \& Estes, M. K. (1995). Detection of Norwalk virus and hepatitis A virus in shellfish tissues with the PCR. Applied and Environmental Microbiology, 61(8), 3014-3018.

4. Block, J. C., \& Schwartzbrod, L. (1989). Viruses in water systems: Detection and identification. New York, USA: VCH Publishers.

5. Brassard, J., Gagné, M. J., Généreux, M., \& Côté, C. (2012). Detection of human food-borne and zoonotic viruses on irrigated, field-grown strawberries. Applied and environmental microbiology, 78(10), 3763-3766.

6. Cavero, P., \& Rodríguez, P. (2008). Producción sostenida de moluscos bivalvos en el Perú: acuicultura y repoblamiento. En: A. Lovatelli, A. Farías, \& Uriarte I. Estado actual del cultivo y manejo de moluscos bivalvos y su proyección futura: factores que afectan su sustentabilidad en América Latina.(pp 209-218). Puerto Montt,Chile: FAO.

7. Da Silva, A. K., Le Saux, J. C., Parnaudeau, S., Pommepuy, M., Elimelech, M., \& Le Guyader, F. S. (2007). Evaluation of removal of noroviruses during wastewater treatment, using real-time reverse transcription-PCR: different behaviors of genogroups I and II. Applied and Environmental Microbiology, 73(24), 7891-7897.

8. De Keuckelaere, A., Li, D., Deliens, B., Stals, A., \& Uyttendaele, M. (2015). Batch testing for noroviruses in frozen raspberries. International journal of food microbiology, 192, 43-50.

9. Fukuda, S., Sasaki, Y., Takao, S., \& Seno, M. (2008). Recombinant norovirus implicated in gastroenteritis outbreaks in Hiroshima Prefecture, Japan. Journal of medical virology, 80(5), 921-928.

10. Gonzaga, V. E., Ramos, M., Maves, R. C., Freeman, R., \& Montgomery, J. M. (2011). Concurrent outbreak of norovirus genotype I and enterotoxigenic Escherichia coli on a US Navy ship following a visit to Lima, Peru. PLoS One, 6(6), e20822.

11. Gregory, J. B., Litaker, R. W., \& Noble, R. T. (2006). Rapid one-step quantitative reverse transcriptase PCR assay with competitive internal positive control for detection of enteroviruses in environmental samples. Applied and environmental microbiology, 72(6), 3960-3967.

12. Hansman, G. S., Doan, L. T. P., Kguyen, T. A., Okitsu, S., Katayama, K., Ogawa, S., ... \& Noda, M. (2004). Detection of norovirus and sapovirus infection among children with gastroenteritis in Ho Chi Minh City, Vietnam. Archives of virology, 149(9), 1673- 
1688.

13. Haramoto, E., Katayama, H., Oguma, K., \& Ohgaki, S. (2005). Application of cation-coated filter method to detection of noroviruses, enteroviruses, adenoviruses, and torque teno viruses in the Tamagawa River in Japan. Applied and environmental microbiology, 71(5), 2403-2411.

14. Hewitt, J., Bell, D., Simmons, G. C., RiveraAban, M., Wolf, S., \& Greening, G. E. (2007). Gastroenteritis outbreak caused by waterborne norovirus at a New Zealand ski resort. Applied and environmental microbiology, 73(24), 7853-7857.

15. Kageyama T., Kojima S., Shinohara M., Uchida K., Fukushi S., Hoshino F.B., ... Katayama, K.(2003).

16. Broadly reactive and highly sensitive assay for Norwalk-like viruses based on real-time quantitative reverse transcription-PCR. J Clin Microbiol, 41, 1548-1557.

17. Katayama, K., Shirato-Horikoshi, H., Kojima, S., Kageyama, T., Oka, T., Hoshino, F. B., ... \& Gojobori, T. (2002). Phylogenetic analysis of the complete genome of 18 Norwalk-like viruses. Virology, 299(2), 225-239.

18. Kitajima, M., Haramoto, E., Phanuwan, C., Katayama, H., \& Ohgaki, S. (2009). Detection of genogroup IV norovirus in wastewater and river water in Japan. Letters in applied microbiology, 49(5), 655-658.

19. Koo, H. L., Ajami, N., Atmar, R. L., \& DuPont, H. L. (2010). Noroviruses: the principal cause of foodborne disease worldwide. Discovery medicine, 10(50), 61 .

20. Le Guyader, F. S., Atmar, R. L., \& Le Pendu, J. (2012). Transmission of viruses through shellfish: when specific ligands come into play. Current opinion in virology, 2(1), 103-110.

21. Lodder, W. J., Vinje, J., Van De Heide, R., de Roda Husman, A. M., Leenen, E. J. T. M., \& Koopmans, M. P. G. (1999). Molecular detection of Norwalklike caliciviruses in sewage. Applied and Environmental Microbiology, 65(12), 5624-5627.

22. Loysi, F., Atmar, R., Guillon, P., Le Cann, P., Pommepuy, M., \& Le Guyader, F. 2005. Real-time RT-PCR for norovirus screening in shellfish. $J$ Virol Methods, 123, 1-7.

23. Marcos, A., Gómez-Sánchez, M., \& Hung, A. (2014). Detección del virus de la Hepatitis A en dos muestras de agua del Río Sechura, usando transcriptasa-reversa PCR en tiempo real. Salud Tecnol Vet, 2(1), 1-6.

24. Maunula, L., Miettinen, I. T., \& Von Bonsdorff, C. H. (2005). Norovirus outbreaks from drinking water. Emerging infectious diseases, 11(11), 1716.

25. Moe, C. L., Christmas, W. A., Echols, L. J., \& Miller, S. E. (2001). Outbreaks of acute gastroenteritis associated with Norwalk-like viruses in campus settings. J Am Coll Health, 50(2), 57-66. doi:10.1080/07448480109596008
26. Ozawa, K., Oka, T., Takeda, N., \& Hansman, G. S. (2007). Norovirus infections in symptomatic and asymptomatic food handlers in Japan. $J$ Clin Microbiol, 45(12), 3996-4005. doi:10.1128/ jcm.01516-07

27. Patel, M. M., Widdowson, M. A., Glass, R. I., Akazawa, K., Vinjé, J., \& Parashar, U. D. (2008). Systematic literature review of role of noroviruses in sporadic gastroenteritis. Emerging infectious diseases, 14(8), 1224.

28. Parashar, U. D., Li, J. F., Cama, R., De Zalia, M., Monroe, S. S., Taylor, D. N., ... \& Glass, R. I. (2004). Human caliciviruses as a cause of severe gastroenteritis in Peruvian children. The Journal of infectious diseases, 190(6), 1088-92.

29. Polo, D., Vilariño, M. L., Manso, C. F., \& Romalde, J. L. (2010). Imported mollusks and dissemination of human enteric viruses. Emerging infectious diseases, 16(6), 1036.

30. Saito, M., Goel-Apaza, S., Espetia, S., Velasquez, D., Cabrera, L., Loli, S., ... \& Zimic, M. Norovirus Working Group in Peru. (2014). Multiple norovirus infections in a birth cohort in a Peruvian periurban community. Clin Infect Dis, 58, 483-491.

31. Sarvikivi, E., Roivainen, M., Maunula, L., Niskanen, T., Korhonen, T., Lappalainen, M., \& Kuusi, M. (2012). Multiple norovirus outbreaks linked to imported frozen raspberries. Epidemiology \& Infection, 140(2), 260-267.

32. Svraka, S., Duizer, E., Vennema, H., de Bruin, E., van der Veer, B., Dorresteijn, B., \& Koopmans, M.(2007). Etiological role of viruses in outbreaks of acute gastroenteritis in The Netherlands from 1994 through 2005. J. Clin Microbiol, 4, 1389-1394

33. Teunis, P. F., Moe, C. L., Liu, P., E Miller, S., Lindesmith, L., Baric, R. S., ... \& Calderon, R. L. (2008). Norwalk virus: how infectious is it?. Journal of medical virology, 80(8), 1468-1476.

34. Unión Europea. (2004). Reglamento (CE) No 853/2004 del parlamento europeo y del consejo de 29 de abril de 2004 por el que se establecen normas especificas de higiene de los alimentos de origen animal. Unión Europea. Recuperado de: https://eurlex.europa.eu/LexUriServ/LexUriServ.do?uri=CON SLEG:2004R0853:20120701:ES:PDF

35. Unión Europea. (2008). Decisión de la comisión de 12 de noviembre de 2008 relativa a las medidas de emergencia para la suspensión de las importaciones de determinados moluscos bivalvos destinados al consumo humano procedentes de Perú (2008/866/ $C E)$. Unión Europea. Recuperado de: https://eur-lex. europa.eu/LexUriServ/LexUriServ.do?uri=OJ:L:200 8:307:0009:0010:ES:PDF

36. Victoria, M., Tort, L. L., García, M., Lizasoain, A., Maya, L., Leite, J. P. G., ... \& Colina, R. (2014). Assessment of gastroenteric viruses from wastewater 
directly discharged into Uruguay River, Uruguay. Food and environmental virology, 6(2), 116-124.

37. Van den Berg, H., Lodder, W., van der Poel, W., Vennema, H., \& de Roda Husman, A. M. (2005). Genetic diversity of noroviruses in raw and treated sewage water. Research in Microbiology, 156(4), 532-540.

38. Westrell, T., Teunis, P., Van den Berg, H., Lodder, W., Ketelaars, H., Stenström, T. A., \& de Roda Husman, A. M. (2006). Short-and long-term variations of norovirus concentrations in the Meuse river during a 2-year study period. Water research, 40(14), 26132620.
39. Wyn-Jones, A. P., Carducci, A., Cook, N., D’Agostino, M., Divizia, M., Fleischer, J., ... \& de RodaHusman, A. M. (2011). Surveillance of adenoviruses and noroviruses in European recreational waters. Water research, 45(3), 1025-1038.

40. Zheng, D. P., Ando, T., Fankhauser, R. L., Beard, R. S., Glass, R. I., \& Monroe, S. S. (2006). Norovirus classification and proposed strain nomenclature. Virology, 346(2), 312-323. 